\title{
Sex differences in the associations of obstructive sleep apnoea with epidemiological factors
}

\begin{abstract}
Tianyi Huang (10 ${ }^{1,2}$, Brian M. Lin ${ }^{1,3}$, Sarah C. Marktt ${ }^{4}$, Meir J. Stampfer ${ }^{1,4}$, Francine Laden ${ }^{4,5}$, Frank B. Hu ${ }^{1,2,4}$, Shelley S. Tworoger ${ }^{4,6}$ and Susan Redline ${ }^{7}$

Affiliations: ${ }^{1}$ Channing Division of Network Medicine, Dept of Medicine, Brigham and Women's Hospital and Harvard Medical School, Boston, MA, USA. ${ }^{2}$ Dept of Nutrition, Harvard T.H. Chan School of Public Health, Boston, MA, USA. ${ }^{3}$ Dept of Otolaryngology, Massachusetts Eye and Ear Infirmary, Boston, MA, USA. ${ }^{4}$ Dept of Epidemiology, Harvard T.H. Chan School of Public Health, Boston, MA, USA. ${ }^{5}$ Dept of Environmental Health, Harvard T.H. Chan School of Public Health, Boston, MA, USA. ${ }^{6}$ Division of Population Science, H. Lee Moffitt Cancer Center and Research Institute, Tampa, FL, USA. ${ }^{7}$ Division of Sleep Medicine, Dept of Medicine, Brigham and Women's Hospital and Harvard Medical School, Boston, MA, USA.
\end{abstract}

Correspondence: Tianyi Huang, 181 Longwood Ave., Rm 353, Boston, MA 02115, USA.

E-mail: tih541amail.harvard.edu

@ERSpublications

Substantial sex differences between OSA and various factors suggest sex-specific mechanisms in OSA http://ow.ly/jDVt30iiOJi

Cite this article as: Huang T, Lin BM, Markt SC, et al. Sex differences in the associations of obstructive sleep apnoea with epidemiological factors. Eur Respir J 2018; 51: 1702421 [https://doi.org/10.1183/ 13993003.02421-2017].

ABSTRACT Despite the well-known male predominance in the prevalence of obstructive sleep apnoea (OSA), sex differences in the associations between OSA and a comprehensive range of epidemiological factors remain less clear.

We examined the prevalence of self-reported OSA in 143326 females (age 48-93 years) from the Nurses' Health Study (NHS) and NHS-II and 22896 males from the Health Professionals Follow-up Study (age 65-101 years) in 2012-2013. Multivariable logistic regression was used to estimate the sex-specific prevalence odds ratios (pOR) and 95\% confidence intervals of OSA by demographic, anthropometric, lifestyle and comorbidity factors.

The overall prevalence of self-reported OSA was $6.4 \%$ in females and $13.8 \%$ in males. After mutual adjustment, the associations of OSA with physical inactivity, hypertension and daytime sleepiness were stronger in females, whereas the associations with waist circumference and witnessed apnoea were stronger in males ( $p$-heterogeneity $<0.01$ ). There were qualitative sex differences in the associations with age (pOR per 5-year increment in females $0.95,95 \%$ CI $0.94-0.96$, and males 1.04, 1.01-1.08; p-heterogeneity $<0.0001$ ) and marital status (pOR for married versus other in females 0.85 , 95\% CI $0.81-0.89$, and males 1.11, 0.99-1.25; p-heterogeneity <0.0001).

Substantial sex differences exist in the associations with various factors, suggesting sex-specific mechanisms in OSA.

This article has supplementary material available from erj.ersjournals.com

Received: Nov 222017 | Accepted after revision: Jan 302018

Conflict of interest: T. Huang has received a grant from the American Heart Association for a postdoctoral fellowship on sleep-related training and research, outside the submitted work. S. Redline has received a grant from NIH for sleep-related research studies, grant support to participate in a multicentre clinical trial and grant support to conduct patient-centred outcomes studies from Jazz Pharmaceuticals, and grant support to participate in a multicentre clinical trial from Beckman Coultier, outside the submitted work.

Support statement: This work was supported by the National Institute of Health (grant number UM1 CA186107, UM1 CA176726, UM1 CA167552). T. Huang is a recipient of the American Heart Association Postdoctoral Fellowship (Founders Affiliate) Award (16POST27480007). Funding information for this article has been deposited with the Crossref Funder Registry.

Copyright CERS 2018 


\section{Introduction}

Obstructive sleep apnoea (OSA) is a highly prevalent sleep disorder associated with increased cardiometabolic morbidity and mortality, with the prevalence being two- to three-fold higher in males than in females [1]. For example, the prevalence of moderate-to-severe OSA (apnoea-hypopnoea index $(\mathrm{AHI}) \geqslant 15$ events. $\mathrm{h}^{-1}$ ) based on the United States National Health and Nutrition Examination Survey (NHANES) population is estimated to be $17 \%$ for males and $9 \%$ for females aged 50-70 years [2]. Studies from other countries reported similar results, although prevalence estimates ranged between $5.3 \%$ and $49.7 \%$ for males and $1.2 \%$ and $23.4 \%$ for females across different populations [1-3]. The underlying reasons for such sex disparities have attracted considerable research interest into potential differences in mechanisms and susceptibility to OSA in males and females.

Anatomical differences in the pharyngeal and upper airway structure may contribute partially to the higher susceptibility to OSA in males compared to females $[4,5]$. The potential favourable influences of female sex hormones (e.g. oestrogen and progesterone) on upper airway collapsibility may further act to result in sex differences in the pathogenesis of OSA $[6,7]$. This is supported by the increase in prevalence of OSA after females undergo menopause [8]. Given that adiposity, particularly upper-body obesity, is a strong risk factor for OSA [9], the well-established sex variations in body composition and fat distribution are considered as another important mechanism modulating sex-dimorphic vulnerability to OSA [10]. Furthermore, presentation and perception of relevant symptoms, such as loud snoring and daytime sleepiness may vary between males and females, leading to differential clinical recognition and diagnosis of OSA $[11,12]$.

However, no study has provided a comprehensive evaluation of the sex-specific associations between OSA and related epidemiological factors. Elucidating sex differences in the associations with sociodemographic, anthropometric, lifestyle and comorbidity risk factors is fundamental for understanding the sex-specific patterns in the development, awareness, clinical presentation and diagnosis of OSA, which has important implications for the targeted prevention and treatment of this disease. Therefore, we compared prevalence of self-reported OSA in three large cohorts of US health professionals, including $\sim 143000$ female nurses and 22000 male health professionals with US national estimates. In addition, we examined the sex-specific associations of self-reported OSA with epidemiological factors, including several novel sociodemographic and lifestyle factors.

\section{Methods}

\section{Study population}

The Nurses' Health Study (NHS) was established in 1976, when 121700 female registered nurses aged 30 55 years completed an initial questionnaire on lifestyle and disease history. The NHS-II is a similar sister cohort initiated in 1989 among 116429 nurses aged 25-42 years. The Health Professionals Follow-up Study (HPFS) enrolled 51529 males aged 40-75 years at baseline in 1986 and comprised dentists, veterinarians, pharmacists, optometrists, osteopathic physicians and podiatrists. Participants in all three cohorts were mailed a follow-up questionnaire every 2 years to prospectively update information on lifestyle factors and disease occurrence. The current analysis included 68451 NHS, 74875 NHS-II and 22896 HPFS participants who responded to a question on OSA in 2012-2013 with concurrent weight information. The study was approved by the institutional review board at the Brigham and Women's Hospital (Boston, MA, USA).

\section{Assessment of self-reported OSA and related symptoms}

Self-reported clinical diagnosis of OSA was assessed in 2012 in NHS and HPFS and in 2013 in NHS-II by the question: "Have you ever been diagnosed with sleep apnoea by a sleep study?" Participants who answered yes were further asked about the year of the diagnosis. Considering the substantial overrepresentation of OSA in the general population compared to central sleep apnoea (CSA), we defined all self-reported cases of sleep apnoea as OSA throughout. Additionally, participants reported whether they snore "every night", "most nights, "a few nights a week", "occasionally" or "almost never". We defined habitual snoring as snoring every night or most nights. Daytime sleepiness was evaluated by the question "On average, how often are your daily activities affected because you are sleepy during the day?" with response categories of "almost every day", "4-6 days per week", "1-3 days per week", "rarely" and "never". Participants who reported $\geqslant 4$ days of sleepiness per week were considered to have daytime sleepiness. In NHS, response to this simple sleepiness assessment was strongly correlated with the Epworth sleepiness scale (Spearman correlation 0.67) [13]. In NHS-II and HPFS, witnessed apnoea was assessed using the question: "Has anyone noticed that you stop breathing during your sleep?"

\section{Validation of self-reported OSA}

To validate self-reported OSA diagnoses, we randomly selected 75 NHS and 75 NHS-II females from those who self-reported an OSA diagnosis and sent them a supplementary questionnaire with a request for 
medical records in November 2016. We asked about diagnostic methods, symptoms leading to the diagnosis, related treatment (surgery, continuous positive airway pressure, oral appliance or supplemental oxygen) and weight change. In addition, we provided a tape for measurement of waist circumference and neck circumference to the nearest $1 / 4$ inch. Several clinical measures were extracted via medical record review, including AHI. Briefly, out of 108 females who returned the questionnaire, all reported having OSA diagnosed by objective monitoring ( $91 \%$ by in-lab polysomnography; online supplementary table S1), which was further confirmed by medical record review. The median (range) AHI was 21 (6-58) events $\cdot \mathrm{h}^{-1}$, and $98 \%$ of cases were classified as obstructive.

\section{Assessment of relevant factors}

We identified a list of established or putative factors related to OSA based on prior literature as well as availability in our cohorts. Participants reported birth date, height and race/ethnicity. Information on weight, smoking, menopausal status, marital status, physical activity and diagnosis of diabetes and hypertension was updated every 2-4 years. We used the data collected concurrently with or closest to the OSA assessment to evaluate cross-sectional associations with OSA prevalence. Participation in various forms of recreational physical activity was quantified by metabolic equivalent task (MET)-h.week ${ }^{-1}$, which integrates both duration and intensity of the activity. Self-reported anthropometric measures [14], menopausal status [15] and physical activity $[16,17]$ have been validated extensively and show excellent reliability.

\section{Statistical analysis}

Sex-specific prevalence estimates of self-reported OSA were calculated according to age $(<50,50-59,60-$ $69,70-79$ and $\geqslant 80$ years) and body mass index (BMI) $(<20.0,20.0-24.9,25.0-29.9,30.0-34.9,35.0-39.9$ and $\left.\geqslant 40.0 \mathrm{~kg} \cdot \mathrm{m}^{-2}\right)$. In addition, a three-way joint analysis by age, sex and BMI was performed. We conducted a similar analysis in females to examine the prevalence by menopausal status within strata of BMI and age. To facilitate comparison of results with national estimates [2], we created broader categories of age $(<50,50-69$ and $\geqslant 70$ years $)$ and BMI $\left(<25.0,25.0-29.9,30.0-39.9\right.$ and $\left.\geqslant 40.0 \mathrm{~kg} \cdot \mathrm{m}^{-2}\right)$ and repeated the analysis.

Logistic regression was used to estimate sex-specific prevalence odds ratios (pOR) and 95\% confidence intervals of self-reported OSA by various factors, as categorised in table 1 . These factors were mutually adjusted in the multivariable model. Linear trend tests were performed for age, BMI, waist circumference and physical activity by using the corresponding factor as a continuous variable. A post hoc analysis with restricted cubic splines was conducted to examine the potential nonlinear association with age in females. We used random-effects meta-analysis to test heterogeneity in the associations by sex.

To assess the impact of undiagnosed OSA on the associations as well as the differences in the associations by disease severity, we further categorised the study population into four groups based on OSA diagnosis and related symptoms: 1) no OSA diagnosis without habitual snoring or daytime sleepiness or both (reference); 2) no diagnosis with habitual snoring plus daytime sleepiness (i.e. high-risk, potentially undiagnosed individuals); 3) OSA diagnosis without daytime sleepiness; and 4) OSA diagnosis with daytime sleepiness (i.e. potentially more severe OSA [18]). Multinomial logistic regression was used to examine whether the sex-specific associations with risk factors differed across these OSA severity categories in a dose-response fashion. All analyses were performed in SAS 9.4 for UNIX (SAS Institute, Cary, NC, USA).

\section{Results}

Out of 143326 females, 9111 (6.4\%) reported a diagnosis of OSA, whereas 3156 (13.8\%) of 22896 males reported the diagnosis. The OSA prevalence increased substantially and monotonically with increasing BMI for both sexes, ranging from $1.5 \%$ to $29.8 \%$ in females and from $8.0 \%$ to $51.2 \%$ in males when BMI increased from $<20$ to $\geqslant 40 \mathrm{~kg} \cdot \mathrm{m}^{-2}$, respectively (figure $1 \mathrm{a}$ ). The age-specific prevalence in females was $5.5 \%$ for $<50$ years, $6.7 \%$ for $50-59$ years, $8.2 \%$ for $60-69$ years, $5.4 \%$ for $70-79$ years and $3.6 \%$ for $\geqslant 80$ years, compared to $13.7 \%$ for $60-69$ years, $14.5 \%$ for $70-79$ years and $12.8 \%$ for $\geqslant 80$ years in males (figure 1b). Stratified jointly by age and BMI, the prevalence in males increased slightly with age within each BMI category (figure 1c), whereas the inverted U-shaped pattern with age was consistently observed for each BMI category among females (figure 1d). The age-, sex- and BMI-specific prevalence estimates of self-reported OSA in these cohorts were remarkably similar to the US estimates of moderate-to-severe $\left(\mathrm{AHI} \geqslant 15\right.$ events $\cdot \mathrm{h}^{-1}$ ) OSA measured using polysomnography (online supplementary table S2) [2].

Overall OSA prevalence was $4.3 \%$ in premenopausal females and $6.4 \%$ in postmenopausal females, with similar increasing patterns of BMI-specific prevalence in both groups (figure 2a). Within each BMI category, the prevalence was consistently higher in postmenopausal than premenopausal females. In 
females aged $<70$ years, the magnitude of the prevalence increase by age was smaller than the increase by menopausal status (figure $2 \mathrm{~b}$ ). However, the prevalence decreased with age among postmenopausal females after the age of 70 years.

In the multivariable logistic analyses mutually adjusted for each factor (table 1), we observed opposite directions in the sex-specific associations of age and marital status with prevalent OSA (p-heterogeneity $<0.0001$ for both). In females, there was an overall downward trend with age (pOR per 5 years $0.95,95 \%$ CI 0.94-0.96), although the adjusted prevalence showed a nonlinear relationship with age (p-nonlinearity $<0.0001$ based on restricted cubic spline), which peaked at age 60-69 years and declined at age $\geqslant 70$ years.

TABLE 1 Sex-specific prevalence odds ratio of self-reported obstructive sleep apnoea (OSA) according to various epidemiological factors $\#$

Odds ratio $(95 \%$ CI)

p for heterogeneity"

Females (NHS and NHS-II) Males (HPFS)

\begin{tabular}{|c|c|c|c|}
\hline \multicolumn{4}{|l|}{ Age years } \\
\hline$<50$ & $0.92(0.72-1.18)$ & & \\
\hline $50-60$ & $0.87(0.82-0.92)$ & & \\
\hline $60-70$ & Referent & Referent & \\
\hline $70-80$ & $0.82(0.77-0.88)$ & $1.12(1.02-1.24)$ & \\
\hline$\geqslant 80$ & $0.61(0.56-0.67)$ & $1.21(1.08-1.35)$ & \\
\hline Per 5 years & $0.95(0.94-0.96)$ & $1.04(1.01-1.08)$ & $<0.0001$ \\
\hline Postmenopausal & $1.43(1.22-1.67)$ & & \\
\hline Non-white & $1.01(0.92-1.11)$ & $1.06(0.88-1.29)$ & 0.65 \\
\hline Married & $0.85(0.81-0.89)$ & $1.11(0.99-1.25)$ & $<0.0001$ \\
\hline \multicolumn{4}{|l|}{ BMI $\mathrm{kg} \cdot \mathrm{m}^{-2}$} \\
\hline$<20$ & $0.79(0.67-0.93)$ & $1.09(0.79-1.49)$ & \\
\hline $20-25$ & Referent & Referent & \\
\hline $25-30$ & $1.51(1.40-1.63)$ & 1.38 (1.24-1.54) & \\
\hline $30-35$ & $2.54(2.34-2.76)$ & $2.08(1.80-2.42)$ & \\
\hline $35-40$ & $3.96(3.61-4.35)$ & $3.48(2.76-4.40)$ & \\
\hline$\geqslant 40$ & $6.30(5.70-6.97)$ & $5.04(3.42-7.41)$ & \\
\hline Per $5 \mathrm{~kg} \cdot \mathrm{m}^{-2}$ & $1.52(1.50-1.55)$ & $1.55(1.46-1.65)$ & 0.56 \\
\hline \multicolumn{4}{|l|}{ Waist circumference $^{+}$} \\
\hline Category 1 & $0.77(0.70-0.85)$ & $0.76(0.67-0.86)$ & \\
\hline Category 2 & Referent & Referent & \\
\hline Category 3 & $1.09(1.00-1.20)$ & $1.16(1.04-1.29)$ & \\
\hline Category 4 & $1.35(1.25-1.47)$ & $1.58(1.37-1.82)$ & \\
\hline Per $5 \mathrm{~cm}$ & $1.08(1.07-1.09)$ & $1.13(1.10-1.16)$ & 0.0009 \\
\hline \multicolumn{4}{|l|}{ Smoking status } \\
\hline Never & Referent & Referent & \\
\hline Past & $1.01(0.96-1.06)$ & $1.01(0.93-1.10)$ & 0.93 \\
\hline Current & $0.78(0.69-0.87)$ & $0.74(0.55-0.98)$ & 0.75 \\
\hline \multicolumn{4}{|l|}{ Physical activity } \\
\hline Quintile 1 & Referent & Referent & \\
\hline Quintile 2 & $0.86(0.81-0.92)$ & $0.91(0.81-1.03)$ & \\
\hline Quintile 3 & $0.88(0.83-0.94)$ & $0.94(0.83-1.06)$ & \\
\hline Quintile 4 & $0.80(0.74-0.86)$ & $0.81(0.71-0.92)$ & \\
\hline Quintile 5 & $0.77(0.71-0.83)$ & $0.83(0.73-0.95)$ & \\
\hline Per 20 MET-h.week ${ }^{-1}$ & $0.94(0.92-0.96)$ & $0.98(0.96-1.00)$ & 0.005 \\
\hline Regular physical exam & $1.58(1.39-1.80)$ & $1.80(1.47-2.21)$ & 0.28 \\
\hline History of hypertension & $1.50(1.42-1.59)$ & $1.30(1.19-1.42)$ & 0.006 \\
\hline History of diabetes & $1.51(1.42-1.60)$ & $1.40(1.25-1.58)$ & 0.29 \\
\hline Witnessed apnoeaf & $20.04(18.16-22.11)$ & 35.52 (32.87-38.37) & $<0.0001$ \\
\hline Habitual snoring & $2.78(2.65-2.92)$ & $2.71(2.50-2.93)$ & 0.55 \\
\hline Daytime sleepiness & $2.45(2.30-2.62)$ & $1.27(1.14-1.42)$ & $<0.0001$ \\
\hline
\end{tabular}

NHS: Nurses' Health Study; HPFS: Health Professionals Follow-up Study; BMI: body mass index; MET:

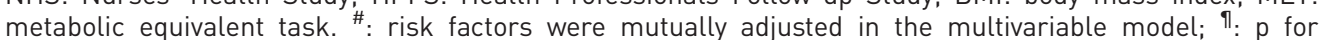
heterogeneity was calculated based on random-effects meta-analysis; ${ }^{+}:$the sex-specific cut-off points of waist circumference were 80,88 and $96 \mathrm{~cm}$ for females and 94,102 and $112 \mathrm{~cm}$ for males; $\S$ : the sex-specific quintile cut-off points of physical activity were 2.9, 8.7, 18.0 and 34.4 MET-h.week ${ }^{-1}$ for females and 14.1, 27.1, 44.1 and 72.3 MET-h.week ${ }^{-1}$ for males; ${ }^{f}$ : among NHS-II and HPFS participants only. 

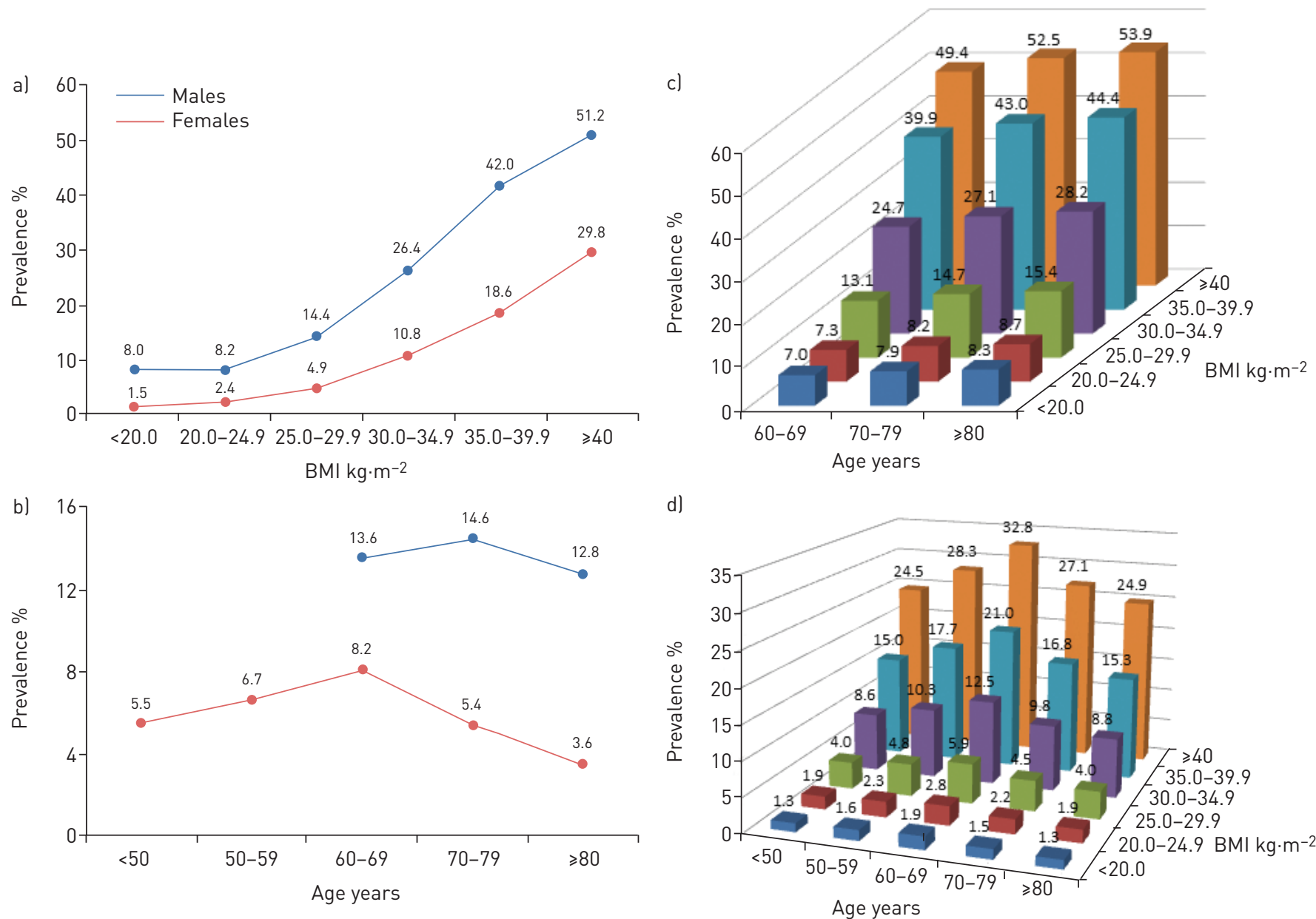

FIGURE 1 Prevalence of self-reported sleep apnoea by a) body mass index (BMI) in males and females; b) age in males and females; and jointly by $\mathrm{BMI}$ and age in $\mathrm{c}$ ) males and d) females.

However, postmenopausal females had higher odds of prevalent OSA compared to premenopausal females (pOR 1.43, 95\% CI 1.22-1.67). In contrast, there was a linear ( $\mathrm{p}$-nonlinearity 0.46 ) positive relationship between OSA prevalence and age in males (pOR per 5 years 1.04, 95\% CI 1.01-1.08). Independent of other factors, being married was associated with lower odds of OSA in females (pOR 0.85, 95\% CI 0.810.89 ), but slightly greater odds in males (pOR 1.11, 95\% CI 0.99-1.25).
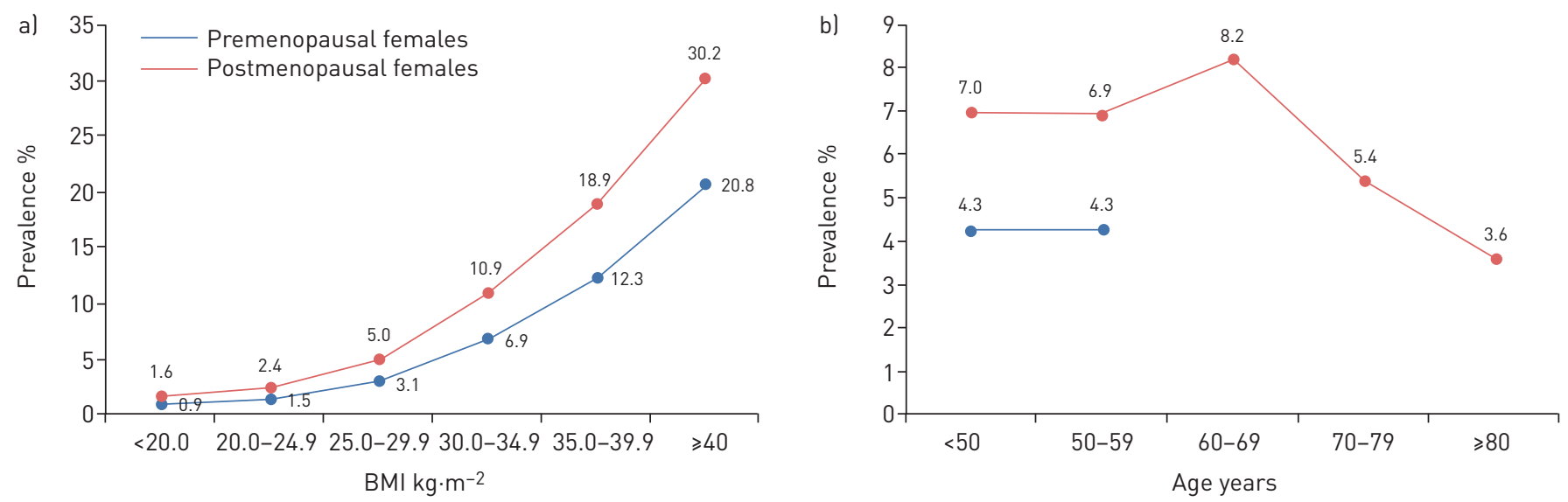

FIGURE 2 Prevalence by a) body mass index (BMI) and b) age in premenopausal and postmenopausal females. 
There were several associations with similar directions but significant differences in magnitude between males and females (table 1). For example, the positive relationship of OSA with waist circumference ( $p$-heterogeneity 0.0009 ) and witnessed apnoea ( $p$-heterogeneity $<0.0001)$ were stronger in males, whereas the positive associations with physical inactivity ( $p$-heterogeneity 0.005 ) and daytime sleepiness ( $\mathrm{p}$-heterogeneity $<0.0001)$ were stronger in females. Compared to males, history of hypertension was more strongly associated with prevalent OSA in females (pOR in females 1.50, 95\% CI 1.42-1.59, and in males 1.30, 1.19-1.42; p-heterogeneity 0.006). A similar pattern was observed for history of diabetes, although the difference was not statistically significant ( $p$-heterogeneity 0.29 ). The sex-specific associations were similar for other risk factors ( $p$-heterogeneity $>0.28$ ), including positive associations with BMI, regular physical exams and habitual snoring and an inverse association with current smoking. No association was seen for past smoking or non-white race in either sex. Sensitivity analyses restricted to those with regular physical exams yielded similar results ( $>93 \%$ of the study participants had regular physical exams; data not shown).

When further dividing participants into four groups according to presence of related symptoms/diagnosis (table 2), the inverse associations with age (i.e. a nonlinear pattern driven by the prevalence decline after age 60-70 years) and physical activity appeared stronger for females with excessive sleepiness regardless of self-reported clinical diagnosis. Similarly, the positive associations with age were only observed for males with sleepiness regardless of diagnosis status. In both sexes, history of hypertension and diabetes exhibited potential dose-response patterns with these categories reflecting OSA severity. For example, the pOR ( $95 \%$ CI) associated with hypertension was 1.29 (1.17-1.42) for high-risk females without self-reported clinical diagnosis, 1.53 (1.43-1.63) for females diagnosed with OSA without sleepiness and 1.70 (1.51-1.92) for females diagnosed with OSA with sleepiness; the corresponding estimates were $1.12(0.95-1.33), 1.29$ $(1.18-1.42)$ and $1.48(1.21-1.82)$ in males.

\section{Discussion}

In this cross-sectional analysis, the age-, sex- and BMI-specific prevalence of self-reported OSA in three large cohorts of health professionals was similar to polysomnography-based estimates of moderate-to-severe OSA in the US, suggesting the validity of self-reported OSA diagnosis among health professionals [2]. In addition, we showed that menopause is a more important determinant of OSA prevalence than age among middle-aged females. Compared to previous polysomnography-based studies, we observed consistent associations of self-reported OSA with established demographic, anthropometric, lifestyle and comorbidity risk factors. Importantly, our comprehensive, sex-specific assessment confirmed the male predominance in OSA prevalence [19] and uncovered intriguing sex differences in the associations of OSA with several important factors, including age, marital status, waist circumference, physical activity, hypertension, sleepiness and witnessed apnoea.

Our results are consistent with prior findings across the world that the OSA prevalence is approximately two- to three-fold higher in males than in females [1]. While the underlying mechanisms are not entirely clear, our results support potential sex differences in the pathogenesis and subsequent health impact of OSA. Given the greater tendency for visceral and hepatic fat accumulation in males compared to females which contributes to higher insulin resistance and inflammation [20], the associations with waist circumference indicate that central obesity and associated metabolic/inflammatory alterations may play more important roles in males than females during OSA development [21]. Conversely, the associations with hypertension and diabetes suggest that females, compared to males, may be at higher risk for cardiometabolic comorbidities associated with OSA. This is consistent with previous studies reporting stronger associations of OSA with incident hypertension, heart failure and endothelial dysfunction in females [22-25]. In line with mounting evidence of the sex differences in the cardiovascular consequences of diabetes [26], it is possible that females with OSA may be more vulnerable to the resulting cardiometabolic risks, while pre-existing metabolic dysfunction may be more likely to predispose males to higher risk for OSA. Considering the cross-sectional nature of this study, additional research is needed to elucidate the sex-specific, bidirectional relationships between OSA and its comorbidities [27].

Trends of age-specific OSA prevalence varied by sex. Previous studies have documented that OSA is more prevalent in postmenopausal than premenopausal females, with age-related prevalence increase reaching the peak by the age of 65 years and levelling off after 65 years [28]. Similarly, we observed an approximately 1.5 -fold higher prevalence in postmenopausal versus premenopausal females, and the highest prevalence among females aged 60-70 years. However, prior studies had limited ability to distinguish between age and menopause. Our analysis reveals that the prevalence increase in females aged $<70$ years is predominantly determined by menopause rather than chronological age. Furthermore, we observed a significant prevalence decrease among females aged $\geqslant 70$ years, which could be due to the "healthy survivor effect", given the strong associations of OSA with comorbidities and premature death 
TABLE 2 Sex-specific prevalence odds ratio for no obstructive sleep apnoea (OSA) diagnosis with habitual snoring plus sleepiness, OSA diagnosis without sleepiness, and OSA diagnosis with sleepiness lversus no OSA diagnosis without habitual snoring or sleepiness) according to epidemiological factors ${ }^{\#}$

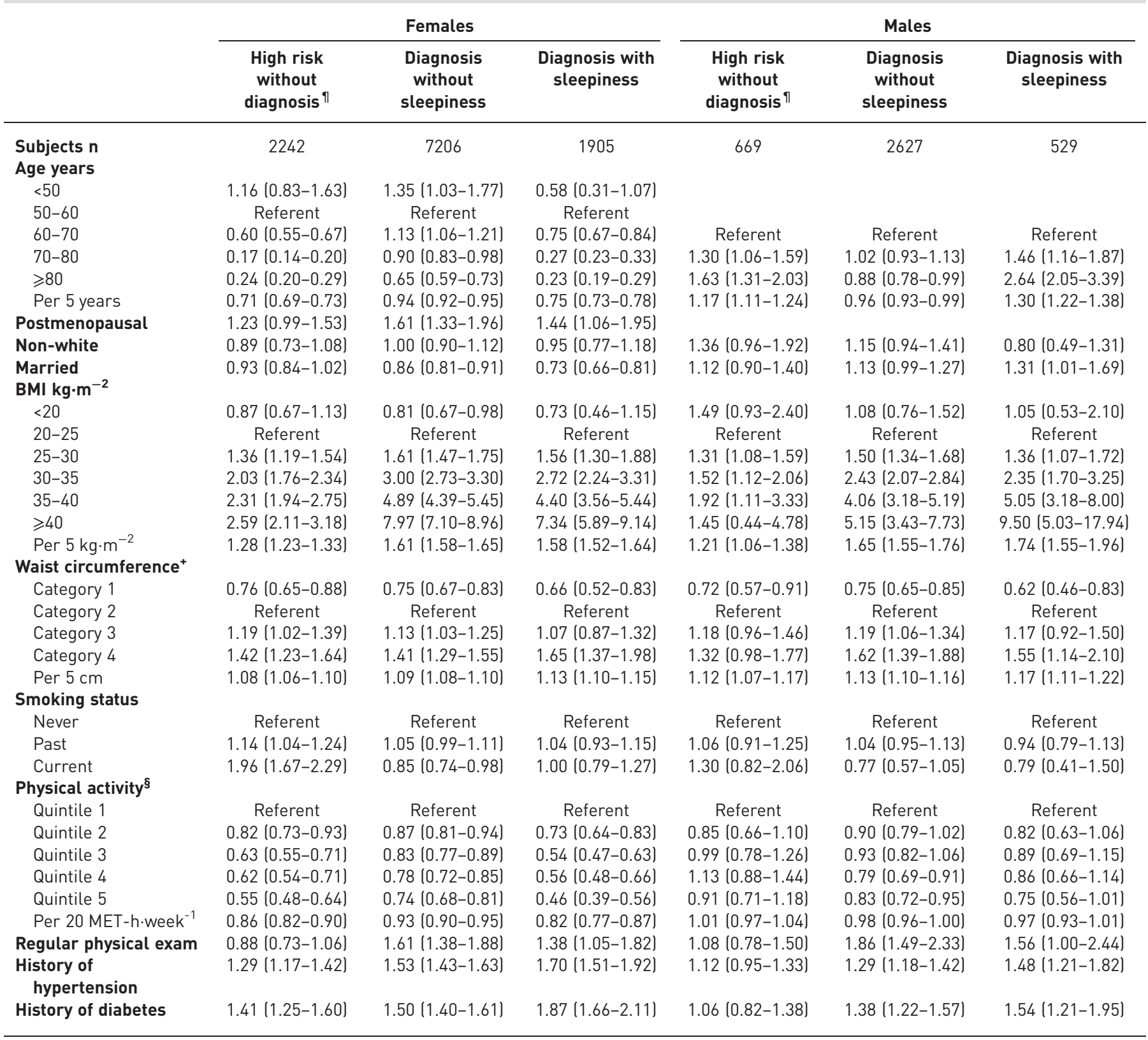

Data are presented as odds ratio $(95 \% \mathrm{CI})$, unless otherwise stated. BMI: body mass index; MET: metabolic equivalent task. "\# : risk factors were mutually adjusted in the multivariable multinominal logistic model; ${ }^{\uparrow}$ : included those who reported habitual snoring plus daytime sleepiness, but did not report clinical diagnosis of sleep apnoea; ${ }^{+}$: the sex-specific cut-off points of waist circumference were 80,88 and $96 \mathrm{~cm}$ for females and 94, 102 and $112 \mathrm{~cm}$ for males; $\S_{\text {; }}$ the sex-specific quintile cut-off points of physical activity were 2.9, 8.7, 18.0 and 34.4 MET-h.week ${ }^{-1}$ for females and 14.1, 27.1, 44.1 and 72.3 MET-h.week ${ }^{-1}$ for males.

[29], particularly if females with OSA have more severe and potentially fatal cardiovascular events [22-24]. Conversely, there was a modest age-related prevalence increase among males after adjustment for BMI. Given the narrower age distribution in males compared to females, further studies are needed to evaluate these associations in younger, middle-aged males.

BMI was one of the strongest correlates of OSA prevalence, with comparable strength of association between males and females, although underweight $\left(\mathrm{BMI}<20 \mathrm{~kg} \cdot \mathrm{m}^{-2}\right)$ was only associated with lower odds of OSA in females, but not in males. Among morbidly obese individuals (BMI $\geqslant 40 \mathrm{~kg} \cdot \mathrm{m}^{-2}$ ), the OSA 
prevalence was as high as $30 \%$ in females and $50 \%$ in males. Of note, the overall OSA prevalence was somewhat lower in the study cohorts (females 6.4\%, males 13.8\%) compared to the US estimates of moderate-to-severe OSA (females 9\%, males 17\%) [2], mainly due to relatively lower prevalence of overweight/obesity in our cohorts versus the general population. Both BMI and waist circumference were independently and strongly associated with OSA, suggesting that biological mechanisms related to general adiposity (e.g. increased systemic inflammation) and body fat deposition (e.g. reduced lung volume due to excess abdominal fat) were both important in OSA.

Results on the relationship between smoking and OSA have been mixed, with most studies reporting positive or no associations [30]. In the Sleep Heart Health Study [31], current smokers were more likely to have lower AHI, similar to the inverse associations between current smoking and OSA consistently observed in males and females of this study. This could be explained by the nicotinic stimulation on upper airway muscle and breathing that reduces OSA [32]. It is also possible that OSA diagnosis may motivate smoking cessation, although we did not observe an association with past smoking. Future studies with a prospective design would be helpful to clarify the relationships between smoking and OSA risk.

A systematic review found that snoring was a less specific indicator for OSA, secondary to nocturnal gasping or choking [33]. In our study, we reported 10-fold larger sex-specific pOR estimates for witnessed apnoea than for snoring. In addition, excessive sleepiness, the major symptom for OSA syndrome, was more strongly associated with prevalent OSA in females than males. Interestingly, females with OSA have often been described as being more fatigued but not as sleepy as males [12, 34]. Our results suggest that female nurses, once they recognised their sleepiness, may be more likely to seek medical care. Furthermore, older females appeared less likely to be diagnosed with OSA with sleepiness, whereas older males were more likely to have the diagnosis with concurrent sleepiness. Understanding sex differences in the age-related recognition and progression of sleepiness (and/or fatigue) symptoms may be beneficial for OSA awareness and diagnosis in the elderly population [35].

Contrary to our hypothesis that marital status, an indicator for bed partners, would be associated with higher odds of OSA diagnosis, we observed differential associations by sex, with significantly lower odds among females. The underlying reasons for this sex difference warrant further investigation, but may be related to threshold for symptom perception/tolerance that is lower for female versus male bed partners [36]. In addition, despite insufficient evidence to recommend screening of OSA in asymptomatic adults [37], our results highlight that regular physical exams may aid in OSA diagnosis for both males and females. Yet we cannot exclude that existing OSA may lead to more regular follow-up medical visits.

Although OSA tends to be underdiagnosed in the general population [38], both the prevalence estimates and the validation study indicate that the self-reported cases among health professionals probably capture moderate-to-severe OSA syndromes, supporting the validity of using self-reported OSA for future studies in these cohorts. However, we should acknowledge that these self-reported diagnoses may not adequately capture mild OSA, which is highly prevalent and often remains unrecognised. A population-based study in Switzerland estimated that up to $83.8 \%$ of males and up to $60.8 \%$ of females aged $40-85$ years had mild-to-severe OSA (AHI $\geqslant 5$ events $\cdot \mathrm{h}^{-1}$ ) [3]. Although whether mild OSA is associated with increased risk of adverse health outcomes has been controversial [39], our symptom-based multinomial analyses, coupled with other emerging evidence [3] support that OSA may exert the biological impact in a dose-response fashion across its severity spectrum. Incorporating symptoms related to OSA, such as sleepiness, may be important to leverage large epidemiological cohorts to study the aetiology of OSA. Importantly, analogous to prehypertension and prediabetes, which are commonly considered as non-cases according to diagnostic criteria despite their potential influence on health, moderate-to-severe OSA captured by self-reported diagnoses will provide valid and critical data for OSA epidemiology.

The strengths of this study include large populations with well-validated epidemiological factors, which enable accurate estimation of prevalence/associations, dissection of age versus menopause effect in females, and well-powered assessment of sex differences. The validity of self-reported OSA has been evaluated and confirmed through three complementary methods, including comparable prevalence to national estimates, expected associations with established factors and confirmation by medical records.

The study has some limitations. First, the cross-sectional design of the study did not allow evaluation of temporality or causality for these sex-specific associations. Second, we did not differentiate OSA versus CSA in the assessment of self-reported diagnosis. Such misclassification probably had minimal impact on the results, given that CSA is much rarer than OSA in the general population [40]. Third, our study populations of predominantly white health professionals may limit the generalisability of the findings. However, the consistent exposure associations compared to prior community-based studies suggest that the aetiology or biological consequences of OSA are unlikely to differ substantially between health professionals and the general population. Fourth, we did not have OSA treatment information, which may 
lead to underestimation of the associations of self-reported OSA with diabetes and hypertension if treatment could effectively alleviate these cardiometabolic comorbidities. Fifth, although we attempted to combine diagnosis status and relevant symptoms as measures for OSA severity and showed consistent severity-response associations with comorbidities, as previously reported with AHI [22, 27], we should be cautious that these proxy measures may not directly correspond to the clinical severity measure determined by AHI or potentially important manifestation of OSA, such as overnight hypoxaemia. Finally, although we did not review medical records to confirm self-reports among male health professionals, we expect similar reliability considering comparable sociodemographic features, health awareness and healthcare access.

In sum, self-reported OSA in US health professionals is reliable, with comparable prevalence to objectively measured moderate-to-severe OSA and expected associations with known epidemiological factors. Significant sex differences were observed for cross-sectional associations of self-reported OSA with age, marital status, abdominal obesity, physical activity, hypertension and OSA-related symptoms. Additional investigation is warranted to confirm the observed sex differences and understand the underlying mechanisms, which could provide more personalised strategies for prevention of OSA and its health consequences. Factors that prompted health professionals to receive a clinical diagnosis of OSA should be identified in future studies, and could be translated to improve OSA underdiagnosis in the general population.

\section{Acknowledgements}

We would like to thank the participants and staff of the Nurses' Health Study, Nurses' Health Study II and Health Professional Follow-up Study for their valuable contributions. The authors assume full responsibility for analyses and interpretation of these data.

\section{References}

1 Lévy P, Kohler M, McNicholas WT, et al. Obstructive sleep apnoea syndrome. Nat Rev Dis Primers 2015; 1: 15015.

2 Peppard PE, Young T, Barnet JH, et al. Increased prevalence of sleep-disordered breathing in adults. Am J Epidemiol 2013; 177: 1006-1014.

3 Heinzer R, Vat S, Marques-Vidal P, et al. Prevalence of sleep-disordered breathing in the general population: the HypnoLaus study. Lancet Respir Med 2015; 3: 310-318.

4 Schellenberg JB, Maislin G, Schwab RJ. Physical findings and the risk for obstructive sleep apnea. The importance of oropharyngeal structures. Am J Respir Crit Care Med 2000; 162: 740-748.

5 Schwab J. Sex differences and sleep apnoea. Thorax 1999; 54: 284-285.

6 Popovic RM, White DP. Upper airway muscle activity in normal women: influence of hormonal status. $J$ Appl Physiol 1998; 84: 1055-1062.

7 Pillar G, Malhotra A, Fogel R, et al. Airway mechanics and ventilation in response to resistive loading during sleep: influence of gender. Am J Respir Crit Care Med 2000; 162: 1627-1632.

8 Bixler EO, Vgontzas AN, Lin HM, et al. Prevalence of sleep-disordered breathing in women: effects of gender. Am J Respir Crit Care Med 2001; 163: 608-613.

9 Mortimore IL, Marshall I, Wraith PK, et al. Neck and total body fat deposition in nonobese and obese patients with sleep apnea compared with that in control subjects. Am J Respir Crit Care Med 1998; 157: 280-283.

10 Ley CJ, Lees B, Stevenson JC. Sex- and menopause-associated changes in body-fat distribution. Am J Clin Nutr 1992; 55: 950-954

11 Redline S, Kump K, Tishler PV, et al. Gender differences in sleep disordered breathing in a community-based sample. Am J Respir Crit Care Med 1994; 149: 722-726.

12 Young T, Hutton R, Finn L, et al. The gender bias in sleep apnea diagnosis. Are women missed because they have different symptoms? Arch Intern Med 1996; 156: 2445-2451.

13 Johns MW. A new method for measuring daytime sleepiness: the Epworth sleepiness scale. Sleep 1991; 14: $540-545$.

14 Rimm EB, Stampfer MJ, Colditz GA, et al. Validity of self-reported waist and hip circumferences in men and women. Epidemiology 1990; 1: 466-473.

15 Colditz GA, Stampfer MJ, Willett WC, et al. Reproducibility and validity of self-reported menopausal status in a prospective cohort study. Am J Epidemiol 1987; 126: 319-325.

16 Wolf AM, Hunter DJ, Colditz GA, et al. Reproducibility and validity of a self-administered physical activity questionnaire. Int J Epidemiol 1994; 23: 991-999.

17 Chasan-Taber S, Rimm EB, Stampfer MJ, et al. Reproducibility and validity of a self-administered physical activity questionnaire for male health professionals. Epidemiology 1996; 7: 81-86.

18 Teodorescu M, Barnet JH, Hagen EW, et al. Association between asthma and risk of developing obstructive sleep apnea. JAMA 2015; 313: 156-164.

19 Block AJ, Boysen PG, Wynne JW, et al. Sleep apnea, hypopnea and oxygen desaturation in normal subjects. A strong male predominance. N Engl J Med 1979; 300: 513-517.

20 Geer EB, Shen W. Gender differences in insulin resistance, body composition, and energy balance. Gend Med 2009; 6: Suppl. 1, 60-75.

21 Simpson L, Mukherjee S, Cooper MN, et al. Sex differences in the association of regional fat distribution with the severity of obstructive sleep apnea. Sleep 2010; 33: 467-474.

22 O'Connor GT, Caffo B, Newman AB, et al. Prospective study of sleep-disordered breathing and hypertension: the Sleep Heart Health Study. Am J Respir Crit Care Med 2009; 179: 1159-1164. 
23 Roca GQ, Redline S, Claggett B, et al. Sex-specific association of sleep apnea severity with subclinical myocardial injury, ventricular hypertrophy, and heart failure risk in a community-dwelling cohort: the Atherosclerosis Risk in Communities-Sleep Heart Health Study. Circulation 2015; 132: 1329-1337.

24 Faulx MD, Larkin EK, Hoit BD, et al. Sex influences endothelial function in sleep-disordered breathing. Sleep 2004; 27: 1113-1120.

25 Mokhlesi B, Ham SA, Gozal D. The effect of sex and age on the comorbidity burden of OSA: an observational analysis from a large nationwide US health claims database. Eur Respir J 2016; 47: 1162-1169.

26 Regensteiner JG, Golden S, Huebschmann AG, et al. Sex differences in the cardiovascular consequences of diabetes mellitus: a scientific statement from the American Heart Association. Circulation 2015; 132: 2424-2447.

27 Aurora RN, Punjabi NM. Obstructive sleep apnoea and type 2 diabetes mellitus: a bidirectional association. Lancet Respir Med 2013; 1: 329-338.

28 Young T, Peppard PE, Gottlieb DJ. Epidemiology of obstructive sleep apnea: a population health perspective. Am J Respir Crit Care Med 2002; 165: 1217-1239.

29 Marshall NS, Wong KKH, Cullen SRJ, et al. Sleep apnea and 20-year follow-up for all-cause mortality, stroke, and cancer incidence and mortality in the Busselton Health Study Cohort. J Clin Sleep Med 2014; 10: 355-362.

30 Krishnan V, Dixon-Williams S, Thornton JD. Where there is smoke...there is sleep apnea: exploring the relationship between smoking and sleep apnea. Chest 2014; 146: 1673-1680.

31 Nieto FJ, Young TB, Lind BK, et al. Association of sleep-disordered breathing, sleep apnea, and hypertension in a large community-based study. Sleep Heart Health Study. JAMA 2000; 283: 1829-1836.

32 Gothe B, Strohl KP, Levin S, et al. Nicotine: a different approach to treatment of obstructive sleep apnea. Chest 1985; 87: 11-17.

33 Myers KA, Mrkobrada M, Simel DL. Does this patient have obstructive sleep apnea?: The Rational Clinical Examination systematic review. JAMA 2013; 310: 731-741.

34 Chervin RD. Sleepiness, fatigue, tiredness, and lack of energy in obstructive sleep apnea. Chest 2000; 118: 372-379.

35 Morrell MJ, Finn L, McMillan A, et al. The impact of ageing and sex on the association between sleepiness and sleep disordered breathing. Eur Respir J 2012; 40: 386-393.

36 Breugelmans JG, Ford DE, Smith PL, et al. Differences in patient and bed partner-assessed quality of life in sleep-disordered breathing. Am J Respir Crit Care Med 2004; 170: 547-552.

37 Jonas DE, Amick HR, Feltner C, et al. Screening for obstructive sleep apnea in adults: evidence report and systematic review for the US Preventive Services Task Force. JAMA 2017; 317: 415-433.

38 Chen X, Wang R, Zee P, et al. Racial/ethnic differences in sleep disturbances: the Multi-Ethnic Study of Atherosclerosis (MESA). Sleep 2015; 38: 877-888.

39 McNicholas WT, Bonsignore MR, Lévy P, et al. Mild obstructive sleep apnoea: clinical relevance and approaches to management. Lancet Respir Med 2016; 4: 826-834.

40 Eckert DJ, Jordan AS, Merchia P, et al. Central sleep apnea: pathophysiology and treatment. Chest 2007; 131: 595-607. 